\title{
Postprandial adropin responses in women co-ingesting green leafy vegetables with a carbohydrate meal: interactions with the sirtuin system
}

\author{
Marietta Sayegh ${ }^{1}$, Andrew Farquharson ${ }^{1}$, Graham Horgan ${ }^{2}$, Viren Ranawana ${ }^{1}$ and \\ Janice E. Drew ${ }^{1}$ \\ ${ }^{1}$ The Rowett Institute and \\ ${ }^{2}$ Biomathematics and Statistics Scotland, University of Aberdeen, Foresterhill, Aberdeen AB25 2ZD
}

Plasma adropin contributes to glucose and lipid homeostasis and cardiovascular system function ${ }^{(1)}$. Adropin levels are influenced by diet $^{(1)}$, but postprandial responses have not been established. The sirtuin system is also implicated in postprandial glucose and lipid homeostasis $^{(2)}$. SIRT1 activity has been shown to increase by fasting and adropin deficiency ${ }^{(3)}$. This study aimed to identify postprandial responses of plasma adropin and association with the sirtuin system following consumption of a carbohydrate meal with or without green leafy vegetables.

Thirty-one women (BMI $24.8-31.1 \mathrm{~kg} / \mathrm{m}^{2}$ ) were recruited to The impact of vegetables on acute glycaemia and glycaemia induced CVD risk in women:metabolic effects and Inter-individual variations (VegGI) study. Anthropometric and metabolic markers were measured in a fasted and postprandial state. Oestradiol, progesterone, follicular stimulating hormone and testosterone were measured. Fasted plasma adropin was measured using an ELISA kit (Cusabio, USA) and $15 \mathrm{~min}, 30 \mathrm{~min}, 45 \mathrm{~min}, 1 \mathrm{~h}, 1.5 \mathrm{~h}, 2 \mathrm{~h}, 3 \mathrm{~h}$ and $4 \mathrm{~h}$ post-consumption of rice, rice with bok choy(RB) or spinach(RS). Total RNA was extracted from blood collected at $0 \mathrm{~h}, 30 \mathrm{~min}, 1$ $\mathrm{h}$ and $2 \mathrm{~h}$ in PAXgene blood RNA tubes (PreAnalytiX GmbH) using a Blood RNA Kit (Qiagen,UK) and quality assessed by Agilent Bioanalyser (Agilent Technologies, UK). Gene expression was measured using the GenomeLab System and a custom designed multiplex assay, the hSIRTNADPlex (25 targets involved in sirtuin signalling). Repeated measures ANOVA was conducted with time, menopausal status and meal as factors. Pearson correlations and Partial Least Squares (PLS) (SIMCA-P+14.0 software) analyses were performed on adropin, gene expression profiles, anthropometric and metabolic markers.

Plasma adropin levels reduced transiently postprandially with time $x$ meal interaction $(p=0.041)$ and time effect $(p<0.01)$. Inter-individual variation was further investigated using PLS of baseline values, indicating that higher fasted adropin was associated with higher sirtuin system gene expression, higher HDL, lower glucose, insulin and HOMA-IR. PLS of baseline and postprandial data indicated a complex pattern of associations for which menopausal status did not appear to be a major factor. Participants with greater postprandial reduction of adropin had higher HDL, lower glucose, insulin and triglycerides. This was supported by significant correlations of adropin, positively with HDL $(0.37-0.59, \mathrm{p}<0.05)$ post-consumption of all test meals, and negatively with insulin postconsumption of rice $(-0.37, \mathrm{p}=0.04)$. Correlations were maintained in analysis of pre-menopausal, but not post-menopausal women. Postprandially, adropin was correlated positively with SIRT4 expression following RB in pre-menopausal $(1 \mathrm{~h}: 0.65, \mathrm{p}=0.04,2 \mathrm{~h}: 0.81$, $\mathrm{p}<0.001)$ but not post-menopausal women.

Postprandial decreases in adropin were time and meal dependent, but with high inter-individual variation. Individuals with lowest levels of adropin were associated with reduced sirtuin system gene expression, higher glucose, insulin and HOMA-IR. Correlations demonstrated complex associations between postprandial adropin reduction, HDL, insulin and SIRT4 gene expression. Plasma adropin may be linked to variation in sirtuin system gene expression, metabolic markers and menopausal status.

\section{References}

1. Drew JE, Reichardt N, Williams, LM et al. (2018) Sci Rep 8, 1.

2. Drew JE, Farquharson AJ, Horgan GW, Duthie SJ and Duthie GG (2014) Mol Nutr Food Res 58, $2066-2079$.

3. Gao S, McMillan RP, Jacas J et al. (2014) Diabetes 63, 3242-3252. 